\title{
Geringfügige Beschäftigung und Segmentation auf innerbetrieblichen Arbeitsmärkten des Einzelhandels
}

\begin{abstract}
Das starke Wachstum der geringfügigen Beschäftigung wirft die Frage auf, welche Auswirkungen diese Entwicklung auf betriebliche Beschäftigungssysteme hat. Am Beispiel von zehn Einzelhandelsunternehmen wird gezeigt, dass der zunehmende Anteil geringfügig Beschäftigter in einigen Unternehmen zu einer vertieften Spaltung in eine Stamm- und eine Randbelegschaft führt. In anderen Unternehmen ist trotz hoher Anteile geringfügiger Beschäftigung eine Spaltung des betrieblichen Arbeitsmarktes nicht festzustellen, denn geringfügig Beschäftigte sind hier Teil der Stammbelegschaft. Die Zunahme der geringfügigen Beschäftigung im Einzelhandel ist durch eine Interessenkoalition zwischen Arbeitsnachfrage und Arbeitsangebot zu erklären: Unternehmen erhalten durch Beschäftigte in kurzen Teilzeitbeschäftigungsverhältnissen ein hohes $\mathrm{Ma} ß$ an numerischer Flexibilität und einige Erwerbsgruppen sind in bestimmten Lebensphasen und unter den institutionellen Rahmenbedingungen von Erwerbsarbeit an einer geringfügigen Beschäftigung interessiert. Ob die Regulierung des geringfügigen Beschäftigungsverhältnisses unter beschäftigungspolitischen Gesichtspunkten sowie unter dem Aspekt einer nur eingeschränkten Partizipation bestimmter Erwerbsgruppen am Erwerbssystem gesellschaftlich wünschenswert ist, bleibt zu diskutieren.
\end{abstract}

\section{$1 \quad$ Einleitung}

Derzeit ist ein starkes Wachstum der geringfügigen Beschäftigung zu beobachten: Nach Angaben der Statistik stieg die Anzahl der geringfügig Beschäftigten im Zeitraum Juni bis Dezember 2003 um 7,4\% auf 6,98 Mio. an, und nach einer Meldung vom April 2004 soll die Zahl im ersten Quartal 2004 weiter auf 7,5 Mio. geringfügige Beschäftigungsverhältnisse angewachsen sein (Minijobzentrale 2003, 2004). Doch bemerkenswert ist nicht nur die Dynamik, mit der die geringfügige Beschäftigung wächst. Auch ihr relativer Anteil von 16,2\% (September 2003) an der gesamten abhängigen Beschäftigung zeigt, dass dieses speziell regulierte Beschäftigungsverhältnis bereits einen festen Platz innerhalb des Gefüges auf dem deutschen Arbeitsmarkt einnimmt (Bundesagentur für Arbeit 2004).

Diese Entwicklung wirft verschiedene Fragen auf: Wer sind diese geringfügig Beschäftigten, für welche Erwerbsgruppen ist die kurze Teilzeitbeschäftigung interessant? Welche Unternehmen nutzen die geringfügige Beschäftigung und welche Position nehmen geringfügig Beschäftigte innerhalb des Arbeitsprozesses ein? Und drittens: Wie verändern sich innerbetriebliche Arbeitsmärkte, wenn kurze Teilzeitbeschäftigung nicht nur sporadisch und am Rande in Unternehmen anzutreffen ist, sondern zunehmend fest ins Kalkül personalstrategischer Entscheidungen einbezogen wird? Diesen Fragen sind wir im Rahmen eines 
Forschungsprojektes nachgegangen, dessen Ergebnisse in diesem Artikel vorgestellt werden. Die forschungsleitende These lautete, dass die Zunahme der geringfügigen Beschäftigung zu einer vertieften Spaltung von Belegschaften, d.h. einer zunehmenden Segmentierung in Stamm- und Randbelegschaften auf betrieblichen Arbeitsmärkten führen muss. Untersuchungsfeld war der Einzelhandel, der sich besonders gut für die Analyse eignet, weil dieser große Dienstleistungssektor zu denjenigen Wirtschaftssektoren gehört, die am häufigsten geringfügige Beschäftigungsverhältnisse anbieten: Bereits jeder vierte Arbeitsplatz im deutschen Einzelhandel ist ein so genannter Minijob (Beschäftigtenstatistik der Bundesagentur für Arbeit 2004). Im Rahmen des Forschungsprojektes, das im Zeitraum Oktober 2003 bis Februar 2004 durchgeführt wurde, wurden Betriebsräte aus zehn Einzelhandelsunternehmen zu Umfang, Struktur aber auch Tätigkeitsfeldern von geringfügig Beschäftigten befragt. Unter den Einzelhandelsunternehmen waren fünf Unternehmen des Lebensmitteleinzelhandels mit unterschiedlichen Vertriebsformen, ein Zentrallager eines Warenhausunternehmens, ein Textilkaufhaus, ein Warenhaus, ein Kaufhaus und ein Drogeriemarktunternehmen.

Der Aufsatz gliedert sich wie folgt: Zunächst werden traditionelle sowie neuere Ansätze der Arbeitsmarktsegmentation vorgestellt, die den theoretischen Bezugsrahmen für die Analyse von betrieblicher Arbeitsmarktsegmentation im Einzelhandel bilden. Es folgt in Kapitel drei eine Einführung in die spezifische Regulierung der geringfügigen Beschäftigung und die Entlohnungssituation von geringfügig Beschäftigten im Einzelhandel. Erwerbsorientierungen von geringfügig Beschäftigten werden im folgenden Kapitel thematisiert. In Kapitel fünf stehen die Segementationslinien im Vordergrund, die in den untersuchten Einzelhandelsunternehmen identifiziert werden konnten. Es zeigt sich, dass eine Beachtung der gesellschaftlichen Rahmenbedingungen unerlässlich ist, um die Segementationen in ihrer Dimension erfassen zu können. Abschließend werden die Ergebnisse zusammengefasst.

\section{Segmentationsansätze als theoretischer Bezugsrahmen}

Die Beschaffenheit von innerbetrieblichen Arbeitsmärkten wird in Ansätzen der Arbeitsmarktsegmentation thematisiert. Als Ausgangs- und Bezugspunkt für die Analyse von Arbeitsmarktsegmentation kann der Ansatzzur Bildung von internen Arbeitsmärkten gewählt werden (Doeringer/Piore 1971). Doeringer und Piore diagnostizierten für die USA in den sechziger und siebziger Jahren eine dualen Wirtschaftsstruktur, die sich in einer Dualisierung des Arbeitsmarktes niederschlug und zu einer Segmentierung des Arbeitsmarktes in ein primäres und ein sekundäres Segment führte. Technologische Entwicklungen und unterschiedliche Nachfrageverhältnisse auf den Gütermärkten wurden als treibende Kräfte der Segmentation identifiziert, die zu Verfestigungen und Starrheiten gegenüber Marktkräften und zur Bildung von abgeschotteten Segmenten auf dem Arbeitsmarkt führten. Dieser Ansatz wurde in der deutschen Diskussion aufgegriffen, und Lutz und Sengenberger (1987) als bekannteste Vertreter entwickelten den „,betriebszentrierten Segmentationsansatz“ (Lutz 1987; Sengenberger 1987). Die spezifischen Bedingungen des bundesdeutschen Berufsbildungssystems wurden von Lutz/Sengenberger als Grundlage für Segmentationen auf dem deutschen Arbeitsmarkt genannt, daher bildet die Qualifikation von Beschäftigten das Abgrenzungskriterium bei der Typisierung von Teilsegmenten. Nach dem Ansatz des betriebsspezifischen Arbeitsmarktes findet sich auf dem dreigeteilten Arbeitsmarkt ein Teilsegement, in dem unspezifische Qualifikationen angeboten und nachgefragt werden. Dieser 
Jederperson-Arbeitsmarkt unterscheidet sich von dem Teilarbeitsmarkt für fachspezifische Qualifikationen (externes Primärsegment) und dem Teilarbeitsmarkt für betriebsspezifische Qualifikationen (internes Primärsegment). Die These von Lutz/Sengenberger lautet, dass die spezifisch deutsche Situation auf dem Güter- und Arbeitsmarkt zu einer Dominanz betriebszentrierter Arbeitsmärkte geführt hat. Betriebszentrierte Arbeitsmärkte seien jedoch nicht in jedem Fall homogen mit Blick auf die Belegschaftsstruktur, sondern könnten sich hinsichtlich des Grades der Internalisierung von Beschäftigung unterscheiden. So könnten Betriebe die Kosten und Risiken der Schließung interner Arbeitsmärkte, die in einer rückläufigen Anpassungsfähigkeit gegenüber externen Einflüssen sowie einer starken Abhängigkeit von der Leistungsfähigkeit der Stammbelegschaften lägen, durch einen kombinierten Einsatz von Stamm- und Randbelegschaften erheblich reduzieren (Sengenberger 1987, 269 f.).

Die Metapher von Stamm- und Randbelegschaften besticht durch ihre Bildhaftigkeit, doch sie ist in zweifacher Hinsicht unterkomplex: Neuere Forschungsergebnisse zur Arbeitsmarktsegmentation in Deutschland belegen, dass zeitlich begrenzte und ,atypische“ Beschäftigungsverhältnisse in der betrieblichen Beschäftigungspolitik nach wie vor eine wichtige Rolle spielen (Köhler u.a. 2004). Doch während in älteren Ansätzen davon ausgegangen wird, dass die Arbeitsplätze in Randbelegschaften vornehmlich diskriminierten Gruppen auf dem Arbeitsmarkt, vor allem Frauen, Migranten oder Unqualifizierten zugewiesen werden, sei nun zu beobachten, dass atypische Beschäftigung auch über die klassischen Problemgruppen des Arbeitsmarktes hinaus verbreitet sei und sich neue Segmentationslinien auch in qualifizierten und hochqualifizierten Belegschaftsstrukturen finden ließen (ebda; Kratzer 2003). Auch der Bezugsrahmen für die Analyse von Segmentation sei zu erweitern: Segmentation fände nicht länger allein auf innerbetrieblichen Arbeitsmärkten statt, sondern die empirische Evidenz zunehmender zwischenbetrieblicher Arbeitsteilung mache es notwendig, Spaltungslinien von Arbeitsmärkten auch betriebsübergreifend und damit bezogen auf Unternehmensnetzwerke zu analysieren (Döhl u.a. 2000; Lehndorff/Voss-Dahm 2004; Rubery u.a.. 2003; für den Einzelhandel: Wirth 1999). Köhler u.a. (2004) schlagen daher vor, die in der deutschen Diskussion eingeführte Typologie von offenen und geschlossenen Beschäftigungssystemen (das entspricht der Terminologie von externen und internen Arbeitsmärkten) um den Typus marktförmig organisierter Beschäftigungssysteme zu erweitern. Dieses Segment des Arbeitsmarktes sei zu charakterisieren 1. durch eine Dominanz von Beschäftigungsverhältnissen, die marktvermittelten Kaufverträgen ähnelten, 2. durch hohe Anteile atypischer Beschäftigung (wie Befristung, Leiharbeit, geringfügige Beschäftigung, freie Mitarbeit) und 3. durch eine lediglich kurze Anlernzeit, die genüge, um die erforderlichen Qualifikationen zu erwerben. Im Unterschied zu den älteren Segmentationsansätzen können in marktförmigen Beschäftigungssystemen alle drei Qualifikationstypen organisiert werden, also berufsfachliche Qualifikationen (z.B. Projektleiter in der Bau- und Softwarebranche), tätigkeitsbasierte Qualifikationen (z.B. freie Dozenten und Journalisten) sowie Jederperson-Qualifikationen (z.B. Verkaufskräfte im Einzelhandel).

Doch nicht nur die Veränderungen von Struktur und Umfang von Randbelegschaften legen eine Überarbeitung der Ansätze zur Arbeitsmarktsegmentation nahe. Rubery (2004) hat in ihrem Entwurf für einen neuen Ansatz der Segmentationstheorie den Einfluss der Arbeitsangebotsseite auf die Bildung von Segmentationslinien auf Arbeitsmärkten integriert mit dem Verweis darauf, dass dieser in den traditionellen Ansätzen nur ungenügend einbezogen worden sei. In der sozialen Organisation von Gesellschaften, eingeschlossen Unterschiede von Klassenzugehörigkeit, Geschlecht, ethnische Herkunft und Qualifikation, sei die Bildung von Segmentationen bzw. von nicht miteinander konkurrierenden Gruppen bereits angelegt. 
Diese externen Segmenationsprozesse würden Segmentationsbildungen auf Arbeitsmärkten stark beeinflussen (Rubery 2004). Mit der stärkeren Beachtung von Strukturen innerhalb des Arbeitsangebots und der Interaktionen zwischen Arbeitsangebot und Arbeitsnachfrageseite rückten - so Rubery - auch die Rahmenbedingungen von Erwerbstätigkeit stärker ins Blickfeld der Analyse. Betriebliche Organisationen blieben zwar zentraler Bezugspunkt der Analyse, doch Arbeitsmarktinstitutionen und soziale Institutionen bekämen ein stärkeres Gewicht, da betriebliche Organisationen in diese eingebettet seien.

Beide Aspekte, die neuen Strukturen in Randbelegschaften sowie die Einbeziehung von Arbeitsangebot und (national)spezifischem Institutionengefüge, werden in der folgenden Analyse von Segmentation in Einzelhandelsunternehmen eine Rolle spielen.

\section{Die gesetzliche Regulierung der geringfügigen Beschäftigung}

Die zum 1. April 2003 in Kraft getretene Regulierung der geringfügigen Beschäftigung unterscheidet sich gegenüber der vorherigen Regelung im Wesentlichen in drei Punkten: Erstens durch die Anhebung des sozialversicherungs- und abgabefreien Arbeitsentgelts von $325 €$ auf $400 €$ (,Minijob“), zweitens durch die Einführung einer Gleitzone im Einkommensbereich zwischen $400 €$ und $800 €$ (,Midijob“), in der die Sozialabgaben gestaffelt ansteigen, sowie drittens durch die Wiedereinführung der Sozialversicherungs- und Abgabenfreiheit für geringfügige Beschäftigungsverhältnisse, die neben einer sozialversicherungspflichtigen Beschäftigung in Nebentätigkeit ausgeübt werden. In diesem Artikel stehen die Minijobs im Zentrum der Betrachtung. Die Regulierung sieht vor, dass die Arbeitgeberseite nicht durch eine Befreiung oder Reduzierung von Sozialversicherungsbeiträgen oder Steuerabgaben begünstigt wird, denn sie entrichten für geringfügig Beschäftigte einen Pauschalbeitrag in Höhe von $12 \%$ an die Rentenversicherung, von $11 \%$ an die Krankenversicherung und 2\% pauschale Steuerbeiträge. Stattdessen ist die Beitrags- und Abgabenbefreiung (und damit die gesellschaftliche Subventionierung dieses Beschäftigungsverhältnisses) an den Einkommensbezug geknüpft mit der Folge, dass das Bruttoeinkommen aus einer geringfügigen Beschäftigung in der Regel dem Nettoeinkommen entspricht. Weil einige Erwerbsgruppen wie z.B. verheiratete Erwerbspersonen mit Ehepartner in voll sozialversicherungspflichtiger Beschäftigung, Rentner, Studierende oder Schüler meist anderweitig sozial abgesichert sind, ist die geringfügige Beschäftigung gerade für diese Erwerbsgruppen interessant (Hauptverband des deutschen Einzelhandels 2003; Weinkopf 2003).

Die Stundenbegrenzung von 15 Wochenstunden, die für die geringfügige Beschäftigung bis April 2003 galt, ist mit der Neuregelung ersatzlos gestrichen worden. Damit ergibt sich die von den geringfügig Beschäftigten zu leistende Stundenzahl unter Beachtung der Einkommensgrenze von maximal $400 €$ indirekt über die Höhe des Stundenlohnes. Die Lohn- und Gehaltstarifverträge im Einzelhandel unterliegen zwar seit einigen Jahren nicht mehr der Allgemeinverbindlichkeit, dennoch kann davon ausgegangen werden, dass sie in der Praxis nach wie vor eine normbindende Wirkung entfalten (Bispinck u.a. 2003). Die Gehaltstarifverträge im Einzelhandel differenzieren nach Tätigkeitsprofil, (in geringerem Maße) über das Alter der Beschäftigten und (in stärkerem Maße) über die Dauer der Erwerbstätigkeit im Handel. Im Angestelltenbereich des Einzelhandels ist das Senioritätsprinzip daher stark verankert. Da die geringfügige Beschäftigung der voll sozialversicherungspflichtigen Beschäftigung rechtlich gleichgestellt ist, differiert der Stundenlohn je nach Eingruppierung 
auch innerhalb der geringfügigen Beschäftigung: Beschäftigte mit langer Erwerbsbindung in den Einzelhandel und kaufmännischer Qualifikation erhalten in Verkaufstätigkeiten (z.B. Gehaltsgruppe 1 im Tarifvertrag des Einzelhandels in NRW) den Stundenlohn des 6. Berufsjahres, das sind 11,94€ Stundenlohn. Unter der Restriktion der Einkommensgrenze des geringfügigen Beschäftigungsverhältnisses von $400 €$ liegt die Stundenzahl für eine Beschäftigte in dieser tariflichen Eingruppierung bei ca. 30 Monatsstunden oder knapp 7 Wochenstunden. Demgegenüber erhält eine unausgebildete Beschäftigte in Verkaufstätigkeiten unter 19 Jahren im ersten Berufsjahr (z.B. eine Schülerin oder Studentin) einen Stundenlohn von 6,95€, der bei einem Einkommen von $400 € \mathrm{zu}$ einer Arbeitsdauer von ca. 50 Monatsstunden führt.

Die im Gehaltstarifvertrag für den Einzelhandel angelegte Lohnspreizung ist erheblich, denn eine junge Beschäftigte, die in der niedrigsten Gehaltsstufe beginnt und im Laufe ihrer Erwerbstätigkeit im Handel eine Ausbildung absolviert, kann über 6 Jahre eine Gehaltssteigerung von $70 \%$ realisieren $(6,95 € \mathrm{zu} 11,94 €$ pro Stunde). Damit wird in der kollektivvertraglichen Regulierung die Tatsache reflektiert, dass Beschäftigte in Abhängigkeit von ihrer Berufserfahrung und Qualifikation unterschiedlich in betriebliche Arbeitsprozesse eingebunden sind: Junge Menschen, die z.B. neben dem Besuch einer Schule oder Universität einer geringfügigen Beschäftigung im Einzelhandel nachgehen, arbeiten auf Einstiegs- und Einfacharbeitsplätzen innerhalb eines engen Aufgabenzuschnitts. Berufserfahrene und fachlich qualifizierte Beschäftigte sind eher breit und damit funktional flexibel einsetzbar, die Leistungsanforderungen sind hier höher und entsprechend höher zu entlohnen.

\section{Erwerbsorientierungen von geringfügig Beschäftigten}

Die These des Forschungsprojekts lautet, dass eine Zunahme der geringfügigen Beschäftigung im Einzelhandel mit einer vertieften Segmentation des Branchenarbeitsmarktes einher geht. In den klassischen Ansätzen zur Segmentation wird betont, dass die treibende Kraft der Segmenation die Nachfrageseite des Arbeitsmarktes sei. Neuere Ansätze, die hier in der Analyse berücksichtigt werden, betonen jedoch, dass diese Strategien auf ein entsprechendes Arbeitsangebot treffen müssen, um sich durchzusetzen. Daher stellt sich die Frage, welcher Einfluss von der Arbeitsangebotsseite auf die Segmentierung von Arbeitsmärkten ausgeht. Welche Erwerbsgruppen bieten ihre Arbeitskraft innerhalb eines geringfügigen Beschäftigungsverhältnisses an? Eine repräsentative Untersuchung der geringfügigen Beschäftigung in Deutschland zeigt ${ }^{1}$, dass der überwiegende Anteil der geringfügig Beschäftigten in andere lebensweltliche Zusammenhänge eingebunden ist. Im Jahre 2001/2002 waren geringfügig Beschäftigte gleichzeitig Schüler (28\%) und Studierende (14\%), Rentner (14\%) und Frauen mit erwerbstätigem Partner, die ca. ein Vierteil aller geringfügig Beschäftigten stellen (Infratest Sozialforschung 2003, 101 f.). Offe (1984, 73 f.) argumentiert, dass Interessengesichtspunkte wie Beschäftigungssicherheit, Karriere und Einkommen für Erwerbsgruppen mit einer Option auf eine arbeitsmarkt-externe Lebensform weniger vordringlich seien als für

1 Die zitierte Untersuchung zur geringfügigen Beschäftigung und Nebenerwerbstätigkeit bezieht sich auf einen Zeitraum, in dem die von 1998 bis 2003 geltende gesetzliche Regelung zur geringfügigen Beschäftigung in Kraft war. Es ist davon auszugehen, dass sich seit dem Zeitpunkt der Untersuchung sowohl Umfang als auch Struktur der geringfügigen Beschäftigung bereits wieder verändert hat. Dennoch wird die Studie hier zitiert, da sie Auskunft über diese Beschäftigungsverhältnisse auf Tätigkeits-, Personen- und Haushaltsebene gibt. Anzumerken ist ferner, dass sich die Untersuchung nicht allein auf den Einzelhandel bezieht. In dem Untersuchungssample üben 12\% aller geringfügig entlohnten Beschäftigten eine Verkaufstätigkeit aus. 
„normale“ Arbeitnehmer. Die Unternehmen wiederum antizipierten diese (unterstellte) Erwerbsorientierung und nähmen an, dass im Falle einer Aufkündigung des Arbeitsverhältnisses mit Personen dieser Erwerbsgruppe nicht mit gravierenden betriebspolitischen Konflikten zu rechnen sei. Aus unterschiedlichen Motiven heraus träfen sich daher die Erwartungen von Unternehmen und Erwerbstätigen. Rubery (2004) spricht in diesem Zusammenhang auch von einem „,beidseitigen Zusammentreffen von Ansprüchen“, das in einer Kanalisierung dieser Erwerbsgruppen in die Randbelegschaften der betrieblichen Arbeitsmärkte mündete.

Doch reicht die Erklärung, dass zugeschriebene bzw. antizipierte gesellschaftliche,,Alternativrollen“ (Offe 1984, 73) die Erwerbsneigung prägen, aus, um zu begründen, dass Erwerbspräferenzen speziell auf die geringfügige Beschäftigung gerichtet sind? Offensichtlich ja, denn der überwiegende Teil (83\%) der westdeutschen geringfügig Beschäftigten arbeitet (unter den gegebenen gesellschaftlichen Rahmenbedingungen) freiwillig in diesem Beschäftigungsverhältnis, darunter 37\%, weil sie sich in Ausbildung befinden, 21\%, weil sie genügend Zeit für andere Dinge haben möchten und 16\%, weil sie sich um Kinder bzw. pflegebedürftige Angehörige kümmern müssen (Infratest Sozialforschung 2003, 122 f.). Doch immerhin würden in Westdeutschland $17 \%$ der geringfügig Beschäftigten gerne länger arbeiten, konnten aber keine geeignete Arbeit finden. Anders ist das Verhältnis zwischen freiwilliger und unfreiwilliger geringfügiger Beschäftigung in Ostdeutschland: 44\% der geringfügig Beschäftigten arbeiten unfreiwillig in diesem Beschäftigungsverhältnis und würden gerne länger arbeiten. Diejenigen, die freiwillig geringfügig beschäftigt sind, geben zu 30\% an, dass sie sich in der Ausbildung befinden und zu 13\%, weil ihnen bei Aufnahme einer voll sozialversicherungspflichtigen Beschäftigung Sozialleistungen oder Unterhaltszahlungen gekürzt würden. Besonders interessant ist, dass in Ostdeutschland häusliche und familiäre Verpflichtungen in Bezug auf die Entscheidung für eine geringfügig entlohnte Tätigkeit nur bei 4\% der befragten Personen eine Rolle spielt. Das deutet daraufhin, dass sich die Erwerbsorientierungen - vor allem von Frauen - in Ost- und Westdeutschland auch 15 Jahre nach der Wiedervereinigung noch stark unterscheiden.

Die deutlichen Unterschiede zwischen Ost- und Westdeutschland sind mit Blick auf die geringfügige Beschäftigung auch im Einzelhandel wiederzufinden: Nach einer vom Institut Arbeit und Technik in Auftrag gegebenen Sonderauswertung der Beschäftigtenstatistik der Bundesagentur für Arbeit lag der Anteil der geringfügigen Beschäftigung im September 2003 je nach Einzelhandelsbranche in Ostdeutschland zwischen 5\% und 10\% unterhalb des Anteils in Westdeutschland. Und auch eine geschlechtsspezifische Verteilung der Beschäftigungsformen ist im Einzelhandel zu erkennen: Drei Viertel aller männlichen Einzelhandelsbeschäftigten arbeiten in Vollzeit, aber auch $18 \%$ arbeiten in einem geringfügigen Beschäftigungsverhältnis. 27\% aller Frauen, die knapp drei Viertel aller Beschäftigten im Einzelhandel stellen, arbeiten in einem geringfügigen Beschäftigungsverhältnis, 32\% der Frauen in einem voll sozialversicherungspflichtigen Teilzeit-Beschäftigungsverhältnis.

Zusammengefasst lässt sich feststellen, dass die Mehrheit der geringfügig Beschäftigten in Deutschland freiwillig in diesem Beschäftigungsverhältnis arbeitet. Dieses Ergebnis quantitativer Untersuchungen deckt sich mit den Aussagen der befragten Betriebsräte. Zwar würden geringfügig Beschäftigte vereinzelt wieder in die voll sozialversicherungspflichtige Beschäftigung wechseln wollen oder müssen. In der Regel jedoch wären geringfügig Beschäftigte mit ihrem derzeitigen Erwerbsstatus zufrieden. Für die Arbeitsnachfrage bedeutet das, dass Unternehmen, die im Rahmen ihrer Personalstrategie auf eine Ausweitung der geringfügigen Beschäftigung setzen, auf ein entsprechendes Arbeitsangebot treffen. 


\section{Segmentation auf betrieblichen Arbeitsmärkten des Einzelhandels}

Welche Personalstrategien verfolgen die zehn Einzelhandelsunternehmen nun im Einzelnen? Die Empirie zeigt, dass das Thema „Flexibilität des Personaleinsatzes“ in alle Unternehmen unternehmenspolitisch einen hohen Stellenwert hat. Doch mit Blick auf die Umsetzung beschreiten die Unternehmen ganz unterschiedliche Wege, so dass innerhalb der zehn untersuchten Unternehmen kein einheitlicher Trend der Ausdifferenzierung von Arbeitszeit und Beschäftigung und insbesondere der Zunahme der geringfügigen Beschäftigung zu verzeichnen ist. Diese Unterschiede schlagen sich auch in unterschiedlich hohen Anteilen der geringfügigen Beschäftigung nieder: Der Anteil dieser Beschäftigungsform variiert in den zehn Einzelhandelsunternehmen zwischen $0 \%$ und 69\%. Innerbetriebliche Arbeitsmärkte in Einzelhandelsunternehmen unterscheiden sich daher in erheblichem Maße mit Blick auf die Beschäftigungsstruktur und damit auch hinsichtlich der Art und Weise, wie der betriebliche Arbeitsprozess organisiert wird.

Die zehn Unternehmen lassen sich mit Blick auf die Beschaffenheit der innerbetrieblichen Arbeitsmärkte in drei Gruppen einteilen. In der ersten Gruppe - darunter ein Warenhaus, das Zentrallager eines Warenhausunternehmens und ein SB-Warenhaus-spielt die geringfügige Beschäftigung keine Rolle, und die innerbetrieblichen Arbeitmärkte sind durch stabile und langfristige Beschäftigungsbeziehungen gekennzeichnet. Qualifizierte Beschäftigte verschaffen den Unternehmen ein hohes Maß an funktionaler Flexibilität, die flankiert wird durch regulierte Arbeitszeitflexibilität (z.B. Jahresarbeitszeitkonten) und eingebettet ist in eine abteilungsübergreifende, teamorientierte Zusammenarbeit. Weil die innerbetrieblichen Arbeitsmärkte hier nicht segmentiert sind, wird in der weiteren Argumentation nicht weiter auf diese Gruppe von Unternehmen Bezug genommen. Den Gegenpol zu diesen drei Unternehmen bilden drei Unternehmen (ein Kaufhaus, zwei Verbrauchermarktunternehmen), die dezidiert die Strategie des segmentierten innerbetrieblichen Arbeitsmarktes verfolgen und in denen der Anteil der geringfügigen Beschäftigung zwischen 35\% und 69\% liegt (Abschnitt 5.1). In der dritten Gruppe von Unternehmen, darunter ein SB-Warenhaus, zwei Verbrauchermarktunternehmen und ein SB-Warenhaus, liegt der Anteil der Minijobber zwischen 15\% und $35 \%$. Trotz der Anteile von geringfügig Beschäftigte kann hier von gespaltenen Arbeitmärkten und einer deutlichen Trennung in Stamm- und Randbelegschaft nicht die Rede sein: Minijobber sind hier Teil der Stammbelegschaft, weil sie in der Regel über ein qualifizierende Ausbildung sowie langjährige Berufserfahrung verfügen. Dadurch sind sie in verschiedenen Einsatzfeldern einsetzbar und verschaffen den Unternehmen zusätzlich ein hohes Maß an numerischer Flexibilität, weil sie punktuell und vor allem zu Spitzenzeiten des betrieblichen Arbeitsanfalls eingesetzt werden (Abschnitt 5.2).

\subsection{Geringfügig Beschäftigte innerhalb von Randbelegschaften}

Die innerbetrieblichen Arbeitsmärkte in drei Unternehmen sind gespalten in zwei Segmente: Stammbeschäftigte bilden die „Anker“ im betrieblichen Ablauf, während die Randbelegschaft ein hohes Maß numerischer Flexibilität zur Bewältigung der schwankenden Kunden- und Warenfrequenzen in die betrieblichen Abläufe einbringt.

Der gespaltene Arbeitsmarkt wird besonders deutlich am Beispiel eines Kaufhauses. Stamm- und Randbelegschaft zeichnen sich dadurch aus, dass sich die Beschäftigungsmerk- 
male innerhalb der Segmente mit Blick auf Qualifikation, Betriebsbindung, Arbeitszeit und die Art des Beschäftigungsverhältnisses stark unterscheiden. Die Segmentation der Belegschaft geht einher mit einer stark funktional differenzierten Arbeitsorganisation, d.h. einzelne Arbeitsbereiche werden organisatorisch bewusst voneinander getrennt und in einzelne abgrenzbare Tätigkeitsgebiete zerlegt. Der Prozess der funktionalen Differenzierung ist begleitet von einer entsprechenden Fragmentierung von Arbeitszeit- und Beschäftigungsstrukturen. Die Stammbelegschaft, die ein Drittel der Belegschaft stellt, arbeitet in Voll- und (langen) Teilzeitarbeitsverhältnissen und ist ausschließlich mit Koordinations- und Steuerungsaufgaben sowohl mit Blick auf die Ware als auch auf das Personal beschäftigt. Zwei Drittel aller Beschäftigten arbeiten in einem geringfügigen Arbeitsverhältnis, darunter viele Schüler und Studierende. Sie wickeln das operative Geschäft ab, d.h. sie kassieren, verräumen Ware und räumen den Verkaufsraum auf. Während die qualifizierte Stammbelegschaft über eine lange und enge Betriebsbindung verfügt, ist unter den geringfügig Beschäftigten eine hohe Fluktuation zu verzeichnen, und eine Orientierung auf eine längerfristige Erwerbstätigkeit in diesem Betrieb ist nicht bis schwach ausgeprägt. Stamm- und Randbelegschaft bilden zwei voneinander getrennte Segmente, denn eine Mobilität zwischen den Segmenten ist nicht zu verzeichnen, und die Arbeits- und Beschäftigungssituation dieser beiden Beschäftigtengruppen unterscheidet sich mit Blick auf alle wesentlichen Merkmale des Arbeitsverhältnisses.

Die Arbeitsplätze der Randbelegschaft sind organisatorisch so in den betrieblichen Arbeitsprozess eingebettet, dass eine kurze Anlernzeit genügt, um abgrenzbare und leicht kontrollierbare Tätigkeiten ausführen zu können. Inhaber dieser Arbeitsplätze müssen daher lediglich über „Jederperson-Qualifikationen“verfügen, um eine geringfügige Beschäftigung in diesem Teilsegment des innerbetrieblichen Arbeitsmarktes aufnehmen zu können. Sofern die Verfügbarkeit dieser Beschäftigtengruppe auf dem externen Arbeitsmarkt gegeben ist, sind die Schwellen für einen schnellen Auf- und Abbau dieser Beschäftigtengruppe für die Unternehmen niedrig. Das Unternehmen kann schnell und flexibel auf Nachfrageschwankungen reagieren und die Belegschaft entsprechend anpassen. Der innerbetriebliche Arbeitsmarkt in diesem Unternehmen zeichnet sich daher durch eine starke Öffnung gegenüber dem externen Arbeitsmarkt aus, denn die Personalstrategie ist auf eine enge Bindung an und einen hohen Austausch mit dem externen Arbeitsmarkt gerichtet.

Auch die beiden anderen Unternehmen, die sich durch segmentierte innerbetriebliche Arbeitsmärkte auszeichnen, zeigen, dass Schüler und Studierende für Einzelhandelsunternehmen offensichtlich eine besonders interessante Erwerbsgruppe darstellen, wenn sie innerhalb einer funktional und zeitlich differenzierten Arbeitsorganisation nach geeigneten Bewerbern für Einfach-Arbeitsplätze in der Randbelegschaft suchen. In beiden Unternehmen werden junge, nicht für die Arbeit im Einzelhandel qualifizierte Beschäftigte vorwiegend am Abend und am Wochenende und daher zeitlich passgenau eingesetzt. In einem Unternehmen wird der Einsatz von Schülern und Studierenden zu diesen gemeinhin als ,unsozial“ bezeichneten Arbeitszeiten ausdrücklich von der betrieblichen Interessenvertretung mit getragen, da dies mit einer Entlastung der Arbeit zu diesen Zeiten für das Stammpersonal verbunden ist. Auch mit Blick auf die Tätigkeiten besteht ein Zusammenhang zwischen der Arbeit der Randbelegschaft und den Arbeitsbedingungen des Stammpersonals: Wenn Schüler und Studierende monotone, einfache Arbeiten (z.B. Ware verräumen, Kassiertätigkeiten) oder körperlich anstrengende Arbeiten (z.B. im Getränkemarkt, Ware aus dem Lager in den Verkaufsraum ziehen) übernehmen, wird das Stammpersonal von diesen Tätigkeiten entlastet.

Doch wird die Arbeit für die jungen Beschäftigten in den Randbelegschaften durch eine 
Politik der dezidierten Verteilung von Flexibilitätslasten bzw. der Zuweisung von monotoner und anstrengender Arbeit nicht prekär? Trifft nicht die alte Kritik zu, dass der Schutz der Stammbeschäftigten mit den Belastungen der Randbelegschaften erkauft wird? Wenngleich diese Kritik nicht gänzlich von der Hand zu weisen ist, ist dennoch anzuführen, dass die Erwerbstätigkeit im Einzelhandel für Schüler und Studierende in der Regel eine Durchgangsoder Übergangsphase darstellt und sie in der Arbeit im Einzelhandel eine Beschäftigung finden, die sich in der Regel mit den durch das Bildungssystem gebundenen Zeiten vereinbaren lässt, die räumlich meist günstig zu erreichen ist und die keine gesonderte Anlern- und Qualifizierungszeit erfordert.

Nur in drei der zehn untersuchten Unternehmen ist zu erkennen, dass junge geringfügig Beschäftigte ein Teilsegment innerhalb innerbetrieblicher Arbeitsmärkte bilden. Die Vermutung ist jedoch, dass dieses Teilsegment im deutschen Einzelhandel in Zukunft anwachsen wird. Drei Trends stützen diese Vermutung. Erstens wurde in einigen Interviews betont, dass Nachwuchskräfte - wegen der oft bemängelten Ausbildungsfähigkeit von Bewerbern auf Ausbildungsplätze - zunehmend von den Einstiegs- und Einfacharbeitsplätzen rekrutiert werden. Jungen Minijobbern wird bei entsprechender Eignung ein Angebot zur Qualifizierung und zum internen Aufstieg unterbreitet. Zweitens zeigt eine Sonderauswertung des Mikrozensus durch das Institut Arbeit und Technik, dass sich die Anzahl der Schüler und Studierenden, die neben ihrer Ausbildung im Einzelhandel arbeiten, zwischen 1995 und 1999 und damit innerhalb von nur fünf Jahren von 31.000 Personen auf 76.000 Personen mehr als verdoppelt hat (Voss-Dahm 2002). Drittens ist diese Entwicklung auch in europäischen Nachbarländern deutlich zu erkennen: So waren im niederländischen Einzelhandel im Jahre 1995 bereits 34\% aller Beschäftigten im Einzelhandel jünger als 23 Jahre alt, im allgemeinen Lebensmitteleinzelhandel sogar $40 \%$ jünger als 21 Jahre (Hoofdbedrijfschap Detailhandel 1996). Ein ähnliches Bild zeigt sich im dänischen Einzelhandel, wo $28 \%$ der Beschäftigten im Jahre 1999 gleichzeitig Schüler und Studenten bzw. 44\% aller Beschäftigten jünger als 25 Jahre alt waren (Boll 2001). Auch in Großbritannien ist die Beschäftigung von Studenten für die Handelsunternehmen zur Normalität geworden. Knapp 1 Mio. Studenten arbeiteten im Jahr 2001 neben ihrem Studium als Teilzeitkraft im Handel, 1984 waren es erst 320.000 (Labour Research 2001). Damit gilt für die drei genannten Ländern, dass die Beschäftigung von Schülern und Studenten längst keine Randerscheinung mehr ist, sondern zum strukturellen Kennzeichen des Branchenarbeitsmarktes geworden ist.

\subsection{Geringfügig Beschäftigte als Teil der Stammbelegschaft}

In vier der zehn Unternehmen kann trotz hoher Anteile geringfügig Beschäftigter nicht von einer Segmentation der innerbetrieblichen Arbeitsmärkte bzw. von einer in Rand- und Stammbeschäftigte gespaltenen Belegschaft gesprochen werden. Vielmehr kommt in den zunehmenden Anteilen von Minijobbern zum Ausdruck, dass sich die Arbeitszeit- und Beschäftigungssituation auch innerhalb des stabilen Segments der qualifizierten und berufserfahrenen Beschäftigten ausdifferenziert. Als wesentlicher Anhaltspunkt für diese These ist anzuführen, dass sich unter den Minijobbern in den vier Unternehmen Beschäftigte mit einer langen Betriebszugehörigkeit befinden. Das sei-so die Interviewpartner-daran zu erkennen, dass Betriebsjubiläen auch unter geringfügig Beschäftigten keine Seltenheit seien.

Zwei Erwerbsgruppen, auf die diese Beschreibungen zutreffen, sind hier besonders zu erwähnen: Zum einen Frauen, die sich nach Ausbildung und voll sozialversicherungspflichtiger Beschäftigung im Einzelhandel in der „Familienphase“ befinden, nun in einem gering- 
fügigen Beschäftigungsverhältnis oft im selben Betrieb weiter arbeiten und an einer geringfügigen Beschäftigung explizit interessiert sind, weil sie dies als bessere Alternative zum vollständigen Verzicht auf Teilhabe am Erwerbsleben sehen. Ähnliches gilt für Rentner, die vor der Verrentung einer sozialversicherungspflichtigen Beschäftigung im Handel nachgingen und nun neben dem Rentenbezug eine geringfügige Beschäftigung ausüben. Innerhalb der Stammbelegschaften dieser Unternehmen finden sich also Beschäftigte, die vormals voll in den innerbetrieblichen Arbeitsmarkt eingebunden waren und nun trotz des Wechsels in das geringfügige Beschäftigungsverhältnis weiterhin dem Segment des berufsfachlichen Arbeitsmarktes zuzurechnen sind. Aus der Perspektive der Beschäftigten und in einer dynamischen Betrachtung deutet der Wechsel des Beschäftigungsverhältnisses auf die Existenz von „Abwärtsmobilität" auf innerbetrieblichen Arbeitsmärkten hin. Diese Abwärtsmobilität aus der voll sozialversicherungspflichtigen und auf Existenzsicherung gerichteten Erwerbstätigkeit in die geringfügige Beschäftigung hinein ist in der Regel verbunden mit einer Veränderung der Lebenssituation. Damit rücken auch die gesellschaftlichen Rahmenbedingungen von Erwerbsarbeit in den Blick. Beschäftigte, die im Laufe ihres Erwerbslebens in der ausgeprägten Teilzeitbranche Einzelhandel einer Tätigkeit unterhalb des Vollzeitstandards nachgingen, haben in Verbindung mit dem ohnehin niedrigen Einkommensniveau in dieser Branche nur geringe Rentenansprüche erwerben können. Die Aufnahme einer geringfügigen Beschäftigung kann für sie im Rentenalter die einzige Möglichkeit sein, um neben dem Rentenbezug die Existenz zu sichern, d.h. Altersarmut zu mildern. Während in diesem Fall die Abwärtsmobilität Ausdruck eines langsamen Ausstiegs aus dem Erwerbsleben ist, markiert die Abwärtsmobilität für viele Frauen in der Mitte des Erwerbslebens eine Zwischenstation: Besonders Frauen in der Familienphase sehen sich bezüglich ihrer Integration in den Arbeitsmarkt Restriktionen ausgesetzt und reagieren mit einer Rücknahme ihres Arbeitsangebots. ${ }^{2}$ Sie haben mit der geringfügigen Beschäftigung einen „Fuß in der Tür“ des Erwerbssystems, streben eine vollständige Integration in den Arbeitsmarkt vorübergehend jedoch nicht an. Die gesellschaftlichen Rahmenbedingungen für Frauenerwerbstätigkeit, die sich u.a. durch mangelnde Kinderbetreuungsmöglichkeiten auszeichnen, wirken daher als Push-Faktoren bei der Abdrängung von Frauen in bestimmten Lebensphasen in die kurze Teilzeitbeschäftigung bzw. in die geringfügige Beschäftigung und werden zur treibenden Kraft für vertikal abwärts gerichtete und mit Stunden- und Einkommensreduzierung verbundene Mobilitätsmuster innerhalb der Stammbelegschaften von Einzelhandelsunternehmen. Hier ergibt sich für betriebliche Akteure ein Gestaltungsfeld: Regelungen sind zu treffen, damit Abwärtsmobilität nicht zur Sackgasse, sondern Aufwärtsmobilität in die voll sozialversicherungspflichtige Beschäftigung durch Regulierung ermöglicht und flankiert wird.

Neben den genannten Push-Faktoren wirken auch Pull-Faktoren bei der Ausdifferenzierung der Arbeitszeit- und Beschäftigungssituation von beruflich orientierten und qualifizierten Einzelhandelsbeschäftigten. Diese liegen im Bereich der von den Unternehmen verfolgten Personalstrategien, die aus verschiedenen Gründen auf die Beschäftigung von qualifizierten Minijobbern gerichtet ist: Wenn Unternehmen - was ihnen offensichtlich gelingt - innerhalb der geringfügigen Beschäftigung qualifiziertes und erfahrenes Personal an sich binden

2 Diese Ausführungen beziehen sich auf die (west)deutsche Situation. Lehndorff (2001) und Jany-Catrice/ Lehndorff (2004) haben in internationalen Vergleichen des Einzelhandelsarbeitsmarktes herausgearbeitet, dass die Erwerbsmuster von Frauen in hohem Maße durch die jeweiligen nationalen Beschäftigungssysteme geformt und beeinflusst werden. So arbeiten Frauen in einigen Ländern, darunter auch in Deutschland, besonders häufig in kurzen Teilzeit-Beschäftigungsverhältnissen, in anderen Ländern, so in Frankreich und in den skandinavischen Ländern, lässt sich die Dominanz von kurzen Beschäftigungsverhältnissen unter weiblichen Einzelhandelsbeschäftigten, vor allem denjenigen in der Familienphase, nicht feststellen. 
können, sind sie in der Lage das in der Vergangenheit aufgebaute „Humankapital“ auch innerhalb der kurzen Teilzeitbeschäftigung weiterhin zu nutzen. Das bedeutet auch, dass mit Zunahme der geringfügigen Beschäftigung keinesfalls in gleichem Maße eine Erosion von berufsfachlichen Standards verbunden sein muss. Zweitens profitieren Unternehmen nicht nur durch die weiterhin mögliche breite Einsetzbarkeit dieser Beschäftigten, sondern sie erhalten darüber hinaus ein noch höheres Maß interner Flexibilität durch die Möglichkeit, Minijobber punktuell zu besonderen Arbeitszeitlagen und/oder zu Spitzenzeiten einzusetzen. Mit der geschilderten Strategie schlagen Unternehmen quasi zwei Fliegen mit einer Klappe, weil sie die funktionale Flexibilität um die numerische Flexibilität ergänzen können. Qualifizierte, geringfügig beschäftigte Verkaufskräfte sind daher zu charakterisieren als Stammbeschäftigte mit einer besonders kurzen Arbeitszeit, die weiterhin in verschiedenen Arbeitsbereichen und punktuell zu besonderen Zeiten ohne gesonderte Anlernzeit eingesetzt werden können (z.B. in der Warenbestellung, an Servicetheken, im Beratungs- und Bedienungsbereich in speziellen Food und Non-Food-Abteilungen) und mit den betrieblichen Abläufen vertraut sind. Sie verfügen über eine starke Orientierung auf die Tätigkeit im Einzelhandel und oft auch auf die Arbeit in einem bestimmten Betrieb. Drittens spricht einiges dafür, dass es in den vier Unternehmen einen Zusammenhang zwischen der Zunahme der geringfügigen Beschäftigung und der ökonomischen Situation des Unternehmens gibt. Insbesondere Betriebe, die rückläufige bzw. stagnierende Umsätze verzeichnen oder auch mit gestiegenen Renditeerwartungen seitens der Unternehmenszentralen konfrontiert sind, nutzen die Regulierung der geringfügigen Beschäftigung als Steigbügel, um eine Reduzierung des Arbeitsstundenvolumens möglichst konfliktfrei durchzusetzen. Wenn Beschäftigte aus der voll sozialversicherungspflichtigen Beschäftigung in die Geringfügigkeit wechseln, wird Arbeitsangebot zurückgedrängt, was sich in einer Reduzierung der Aufwendungen für Personal niederschlägt. Die Personaldecke wird zwar ausgedünnt (und es kommt zur Leistungsverdichtung der verbleibenden Belegschaft), doch die funktional flexibel und zeitlich punktuell einsetzbar geringfügig Beschäftigten können durch ihre Pufferfunktion einen Teil der entstehenden „Löcher“ in der betrieblichen Organisation wieder auffangen.

\subsection{Interessenkoalition zwischen Unternehmen und Beschäftigten innerhalb der geringfügigen Beschäftigung}

Fakt ist, dass die geringfügige Beschäftigung eine starke Rolle auf dem Branchenarbeitsmarkt des Einzelhandels einnimmt. In der Analyse der damit verbundenen Veränderungen für die Funktionsweise innerbetrieblicher Arbeitsmärkte erwies sich der Segmentationsansatzals ein brauchbarer theoretischer Bezugsrahmen: In einigen Einzelhandelsunternehmen bilden die Stamm- und Randbelegschaft jeweils voneinander getrennte Segmente auf den innerbetrieblichen Arbeitsmärkten, es gibt keine Mobilität zwischen den Segmenten und die Beschäftigungsbedingungen innerhalb der Segmente unterscheiden sich hinsichtlich aller wesentlichen Merkmale. Doch andere Unternehmensbeispiele zeigen, dass traditionelle Segmentationsansätze angesichts veränderter arbeitsmarktpolitischer Rahmenbedingungen und heterogener werdenden Erwerbsorientierungen in ihrem Erklärungsgehalt an Grenzen stoßen, weil sie die institutionellen Rahmenbedingungen von Erwerbsarbeit, die das Arbeitsangebot in starkem Maße beeinflussen, konzeptionell nur unzureichend einbeziehen. In diesem Artikel wurden die Motive, die das Interesse bestimmter Erwerbsgruppen an einer geringfügigen Beschäftigung begründen können, in die Analyse mit einbezogen. Dadurch zeigte sich, dass Einzelhan- 
delsbeschäftigte in so genannten atypischen Beschäftigungsverhältnissen wie der geringfügigen Beschäftigung trotz des Wechsels aus einer voll sozialversicherungspflichtigen Beschäftigung im stabilen Teilsegment des innerbetrieblichen Arbeitsmarktes verbleiben können und innerbetriebliche Mobilität durchaus den Wechsel des Beschäftigungsverhältnisses mit einschließen kann.

Zusammenfassend lässt sich feststellen, dass Nachfrage und Angebot auf dem Arbeitsmarkt die gegenwärtige Entwicklung auf dem Branchenarbeitsmarkt wechselseitig befördern: Unternehmen, die im Rahmen ihrer Personalstrategie auf eine mehr oder weniger starke Fragmentierung der Beschäftigungsverhältnisse setzen, treffen auf dem Arbeitsmarkt derzeit auf ein Arbeitsangebot, das seine Arbeitskraft nur in eingeschränktem Maße anbietet und mit der Erwerbstätigkeit weder das Ziel des beruflichen Aufstiegs, noch der Existenzsicherung verbindet. Erwerbspersonen wiederum, die nur partiell erwerbsorientiert sind, erhalten Rückenwind bei der Durchsetzung ihrer (aus den gesellschaftlichen Rahmenbedingungen abgeleiteten) Erwerbspräferenzen durch die entsprechenden Personalstrategien der Unternehmen. Anders formuliert kommt es zu Interessenkoalitionen zwischen Angebots- und Nachfrageseite des Arbeitsmarktes, die als Triebkräfte der Zunahme des geringfügigen Beschäftigungsverhältnisses wirken und durch institutionelle Rahmenbedingungen gestützt werden.

\section{Schlussfolgerungen}

Die empirischen Befunde aus dem Einzelhandel zeigen, dass unterschiedliche Erwerbsgruppen in geringfügigen Beschäftigungsverhältnissen arbeiten. Geringfügig Beschäftigte im Einzelhandel bilden also kein in sich homogenes Segment. Das bedeutet auch, dass positive bzw. negative Merkmale mit Blick auf die Arbeitszeit, die Stabilität des Beschäftigungsverhältnisses oder auf die soziale und rechtliche Absicherung auf diesem speziellen Beschäftigungsverhältnis nicht eindeutig kumulieren. Eine Bewertung der Qualität eines Arbeitsverhältnisses ist offensichtlich immer weniger allein mit Blick auf die Art des Beschäftigungsverhältnisses vorzunehmen und eindeutige Spaltungslinien, die eine Unterscheidung von „Gewinnern“ und „Verlierern“ermöglichen, sind in den Einzelhandelsunternehmen unter Bezugnahme auf die Art des Beschäftigungsverhältnisses nicht zu erkennen. Vielmehr zeigt sich, dass die feingliedrigen Segmentierungen auf innerbetrieblichen Arbeitsmärkten durch vielfältige Verschränkungen von Sicherheiten und Risiken innerhalb der Beschäftigungsverhältnisse gekennzeichnet sind. Diese Verschränkungen ergeben sich durch die unterschiedlichen zeitlichen und inhaltlichen Anforderungen der Unternehmen an verschiedene Beschäftigtengruppen, durch die unterschiedliche Einbindung von Beschäftigungsverhältnissen in das staatliche Sozialversicherungs- und Steuersystem und durch die heterogenen Erwerbsorientierungen von Erwerbsgruppen in ihrer jeweiligen Lebenssituation.

Die Entwicklungen auf den innerbetrieblichen Arbeitsmärkten haben auch Implikationen für die Makroebene: Erstens fördert die gesetzliche Regulierung der geringfügigen Beschäftigung, die aufgrund der reduzierten Steuer- und Abgabenpflicht als subventionierte Beschäftigung zu bezeichnen ist, offensichtlich nicht die Reintegration von Arbeitslosen oder Transfereinkommensbeziehern in den Branchenarbeitsmarkt des Einzelhandels. Das von der Politik dezidiert verfolgte Ziel, mit der Neuregelung des geringfügigen Beschäftigungsverhältnisses die Arbeitslosigkeit zu reduzieren, wurde damit nicht erreicht. Zweitens ist zu 
diskutieren, ob die Verschränkung von Ausbildung und Erwerbsarbeit bei jungen Menschen unter dem Gesichtspunkt der Qualitätssicherung von Bildungsstandards gesellschaftlich erwünscht ist. Und drittens ist die Frage zu stellen, ob Frauenerwerbstätigkeit, die sich in Deutschland vielfach unter den Bedingung einer Mitversicherung in der Kranken- und Pflegeversicherung, durch Ehegattensplitting im Steuersystem und durch mangelnde Kinderbetreuung gestaltet, langfristig wirklich weiterhin in die kurze Teilzeit abgedrängt und kanalisiert werden soll (Bosch 2001; Wagner 2000). Solange diese Rahmenbedingungen die Frauenerwerbstätigkeit flankieren, ist es nicht verwunderlich, dass die Erwerbsorientierungen vieler (westdeutscher) Frauen auf eine „Arbeit in Häppchen“ ausgerichtet sind und mit Blick auf ihre Position auf innerbetrieblichen Arbeitsmärkten „Abwärtsspiralen“ in Gang setzen. Die derzeitige Regulierung der geringfügigen Beschäftigung bedeutet gerade für Frauen zumindest kurzfristig - einen hohen Anreiz, die Orientierung auf eine eigenständige Erwerbstätigkeit hinten anzustellen und bricht daher mit dem Leitbild einer zwischen den Geschlechtern gleichberechtigten Teilnahme am Erwerbsleben.

\section{Literatur}

Bispinck, Reinhard, Johannes Kirsch, Claus Schäfer (2003): Mindeststandards für Arbeits- und Einkommensbedingungen und Tarifsystem. Projekt im Auftrag des Ministeriums für Wirtschaft und Arbeit des Landes Nordrhein-Westfalen. Düsseldorf

Boll, Joachim (2001): The ,Transitional Workforce': A Source of Time Flexibility in Danish Retail Trade. 8th International Symposium on Working Time, Amsterdam

Bosch, Gerhard (2001): Konturen eines neuen Normalarbeitsverhältnisses; in: WSI-Mitteilungen 4, 219-230

Bundesagentur für Arbeit (2004): Beschäftigtenstatistik. http://www.pub.arbeitsamt.de/hst/services/ statistik/detail/b.html am 25.5.2004

Doeringer, Peter B., Michael J. Piore (1971): Internal Labor Markets and Manpower Analysis. Lexington

Döhl, Volker, Nick Kratzer, Dieter Sauer (2000): Krise der NormalArbeit(s)politik. Entgrenzung von Arbeit - neue Anforderungen an Arbeitspolitik; in: WSI-Mitteilungen 1, 5-17

Hauptverband des Deutschen Einzelhandels (2003): Geringfügige Beschäftigung und GleitzonenBeschäftigung. Erläuterungen der gesetzlichen Regelungen. Berlin

Hoofdbedrijfschap Detailhandel (1996): Arbeidsmarkt in de detailhandel 1996/1997. Den Haag

Infratest Sozialforschung (2003): Geringfügige Beschäftigung und Nebenerwerbstätigkeiten in Deutschland 2001/2002. München

Jany-Catrice, Florence, Steffen Lehndorff (2004): Who works for the retail trade? Working Conditions and Labour Markets in the European Retail Trade; in: Gerhard Bosch, Steffen Lehndorff (Hg.): Working in the service sector - a tale from different Worlds. London/New York (im Erscheinen)

Köhler, Christoph, Olaf Struck, Anja Bultemeier (2004): Geschlossene, offene und marktförmige Beschäftigungssysteme. Überlegungen zu einer empiriegeleiteten Typologie. SFB 580 Mitteilungen. Jena/Halle

Kratzer, Nick (2003): Arbeitskraft in Entgrenzung. Grenzenlose Anforderungen, erweiterte Spielräume, begrenzte Ressourcen. Berlin

Labour Research (2001): A student is not just for Christmas; in: Labour Research 90, 12, 12-14

Lehndorff, Steffen (2001): Der europäische Einzelhandel und die Regulierung von Arbeitsmärkten; in: Hedwig Rudolph (Hg.): Aldi oder Arkaden? Unternehmen und Arbeit im europäischen Einzelhandel. Berlin, 131-156 
Lehndorff, Steffen, Dorothea Voss-Dahm (2004): The delegation of uncertainty: flexibility and the role of the market in service work; in: Gerhard Bosch, Steffen Lehndorff (Hg.): Working in the service sector - a tale from different worlds. London/New York (im Erscheinen)

Lutz, Burkhart (1987): Arbeitsmarktstruktur und betriebliche Arbeitskräftestrategie. Eine theoretischhistorische Skizze zur Entstehung betriebszentrierter Arbeitsmarktsegmentation. Frankfurt/New York

Minijobzentrale (2003): Aktuelle Entwicklungen im Bereich der geringfügigen Beschäftigung. Ausgabe $3 / 2003$. Essen

Minijobzentrale (2004): Minijobzentrale feiert Geburtstag. Pressemeldung vom 01.04.2004. http:// www.minijobzentrale.de/5_Presse/index.html am 25.04.04

Offe, Claus (1984): „Arbeitsgesellschaft“: Strukturprobleme und Zukunftsperspektiven. Frankfurt/ New York

Rubery, Jill u.a. (2003): Inter-organizational Relations and Employment in a Multi-employer Environment; in: British Journal of Industrial Relations 41, 2, 265-289

Rubery, Jill (2004): The shaping of work and working time in the service sector: a segmentation approach; in: Gerhard Bosch, Steffen Lehndorff (Hg.): Working in the service sector - a tale from different worlds. London/New York (im Erscheinen)

Sengenberger, Werner (1987): Struktur und Funktionsweise von Arbeitsmärkten. Die Bundesrepublik Deutschland im internationalen Vergleich. Frankfurt/New York

Voss-Dahm, Dorothea (2002): Erwerbstätigkeit von SchülerInnen und Studierenden nimmt zu. IATReport 2002-06. Gelsenkirchen

Wagner, Alexandra (2000): Krise der Normalarbeitsverhältnisse: Über eine konfuse Debatte und ihre politische Interessenorientierung; in: Claus Schäfer (Hg.): Geringere Löhne - mehr Beschäftigung? Niedriglohn-Politik. Hamburg, 200-246

Weinkopf, Claudia (2003): Mini- und Midijobs. Neue Impulse für den Arbeitsmarkt?; in: Berufsbildung, 83, 23-29

Wirth, Carsten (1999): Unternehmensvernetzung, Externalisierung von Arbeit und industrielle Beziehungen. München/Mering

Anschrift der Verfasserin:

Dipl. Soz. Ök. Dorothea Voss-Dahm

Institut Arbeit und Technik (IAT)

Forschungsschwerpunkt Arbeitszeit und Arbeitsorganisation

Munscheidstr. 14

D-45886 Gelsenkirchen

\section{Schlagwörter: Arbeitsmarkt, Arbeitsorganisation, Arbeitszeit}

